\title{
Flexible barrier materials for protection against electromagnetic fields and their characterization ${ }^{\star}$
}

\author{
Maciej Jaroszewski ${ }^{\mathrm{a}}$ \\ Wroclaw University of Technology, Faculty of Electrical Engineering, Wybrzeze Wyspianskiego 27, 50-370 Wroclaw, Poland
}

Received 30 June 2015 / Received in final form 1st September 2015

Published online 14 October 2015

(C) The Author(s) 2015. This article is published with open access at Springerlink.com

\begin{abstract}
Composite materials for electromagnetic shielding can be manufactured as textiles using conductive yarns and textiles with conductivity obtained by various finishing processes on textile surfaces. The EM shielding effectiveness of fabrics are improved by lowering its conductivity using different methods and materials. An alternative is the usage of new light shielding materials in the form of metallized nonwoven fabrics or textiles. Their advantages are: a general availability on the market, a low price, good mechanical properties (strength, elasticity) and resistance to the environmental conditions. The composite anisotropic materials with a sandwich structure constituting of materials with different spatial orientations of fibers allow one to achieve relatively high and constant values of the shielding effectiveness which, together with the materials' mechanical properties, leads to a wide range of applicability in various disciplines of modern technology. This article is devoted to innovative flexible materials shielding electromagnetic field. The results of the PEM shielding effectiveness obtained for the polypropylene (PP) nonwoven fabrics metallized by pulsed magnetron sputtering are presented.
\end{abstract}

\section{Introduction}

Currently, one of the most extensively developing field of applications of composite materials, is engineering of materials used for shielding from electromagnetic fields $[1-5]$. The increasing interest in many fields of technology are recorded in the application of textiles with intentionally modified surface properties [6,7]. Due to good mechanical properties and resistance to environmental impacts, exceptional importance acquire textile products based on polymers. Composites of polymeric fabric materials with thin plasma layer can be used as shield for protection of people or electronic and electric power devices against of disadvantageous impact of electromagnetic (EM) fields $[8,9]$.

Materials used in the technique of shielding EM fields must have a sufficiently high value of shielding effectiveness factor (SE), defined as:

$$
S E=10 \log \frac{P_{0}}{P_{t}}=20 \log \frac{E_{0}}{E_{t}}=20 \log \frac{H_{0}}{H_{t}}
$$

where: $P_{0}, P_{t}$ - surface power density of the incident EM wave $P_{0}$ and transmitted through screen $P_{t} ; E_{0}, E_{t}$ - the electric field of the incident wave and transmitted through the screen, respectively; $H_{0}, H_{t}$ - the magnetic field of

\footnotetext{
* Contribution to the Topical Issue "Materials for Dielectric Applications", edited by Maciej Jaroszewski and Sabu Thomas.

${ }^{a}$ e-mail: maciej.jaroszewski@pwr.edu.pl
}

the incident wave and transmitted through the screen, respectively.

Considering the case of a homogeneous flat screen, the total shielding effectiveness is the sum of shielding effectiveness by phenomena from: reflection (r), absorption (a) and multiple reflections and transmissions (tmr):

$$
S E=S E_{\mathrm{r}}+S E_{\mathrm{a}}+S E_{\mathrm{tmr}} .
$$

The ability of composite materials for shielding the electromagnetic field, is intrinsically linked to the dielectric parameters of the material [10-12]:

$$
S E=20 \log \frac{Z_{0}}{4 Z_{s}}+20 \log e^{d / \delta}+20 \log \left|1-e^{-2 d / \delta}\right|,
$$

where: $Z_{0}$ - wave impedance of vacuum; $Z_{s}$ - wave impedance shielding material; $d$ - thickness of the screen; $\delta$ - the depth of penetration of the electromagnetic wave.

For the screen made of a highly conductive material and a thickness substantially greater than the depth of penetration:

$$
d \gg \delta=\frac{1}{\sqrt{\pi f \mu \sigma}},
$$

where: $f$ - frequency of the EM field; $\mu$ - magnetic permeability; $\sigma$ - conductivity.

Screen attenuation depends only on the return loss and absorption:

$S E=S E_{\mathrm{r}}+S E_{\mathrm{a}}=\left(39.5+10 \log \frac{\sigma}{2 \pi f \mu}\right)+8.7 \sqrt{\pi f \mu \sigma}$. 
For the largest values of shielding effectiveness, we may use both phenomena. However, the main intention of the presented solution was to obtain screens reflecting electromagnetic waves.

As you can see from the above equation the surface reflecting the wave must be electrically conductive. In the case of shields produced from a foil, metal sheets, or shielding meshes, it does not constitute a major technical problem to achieve a large value (above $70 \mathrm{~dB}$ ) of SE coefficient for the electrical component of the EM field. However, such shields are expensive, heavy, and not applicable everywhere. These disadvantages do not have composite materials. Composite materials, in contrast to solid metallic screens, are characterized by good mechanical properties (low weight, high flexibility), resistance to environmental conditions (temperature, UV radiation, corrosion, ... ), stability and constancy of the parameters during the operation and low production costs.

Composite materials for electromagnetic shielding can be manufactured as textiles using conductive yarns. An alternative is the usage of new light shielding materials in the form of plasma coated polypropylene nonwoven fabrics.

\section{Polymeric textile based composite with conducting thin plasma layer for EM screening}

Nowadays, the most often used method for obtaining different kinds of layers is magnetron sputtering (glow discharge, plasmatic). This method is based on bombarding of surface of the sputtering electrode (the target) with high energetic ions of working gases. Ions knock out atoms or molecules of material from the target, which next are deposited on substrates. This way metallic layers, dielectric semiconductor layers and high-melting layers can be deposited. Sputtering devices using an electrical and a magnetic field are called magnetron or plasmatic guns. In electrical and magnetic fields, ions from working gases obtain very high kinetic energy, and to the layers can be deposited at a very high speed which for metals is a few $\mu \mathrm{m} / \mathrm{min}$ and for dielectric layers is in the order of $10-100 \mathrm{~nm} / \mathrm{min}$. Such high speeds of putting on layers are not available in other methods. Additionally during a process of sputtering, plasma cleans and activates surface of substrates. Therefore with magnetron methods one can do covering of materials such as: PTFE (polytetrafluoroethylene) or PP (polypropylene), which cannot be transformed by covering with other methods, because of their surface properties. Nonwoven fabrics were covered with carbon, various metals and oxide layers.

Microscopic surface morphology studies have shown that metallic $(\mathrm{Me})$ layer formed solid and continuous layer on the surface of the nonwoven (Figs. 1a, 1d, and 1e). It is noteworthy, that the metal layer not only coverage the surface of nonwoven, but also internal fibers (Fig. 1b). Increasing power emitted on sputtered electrode results in covering of welding places of nonwoven fabric, increases

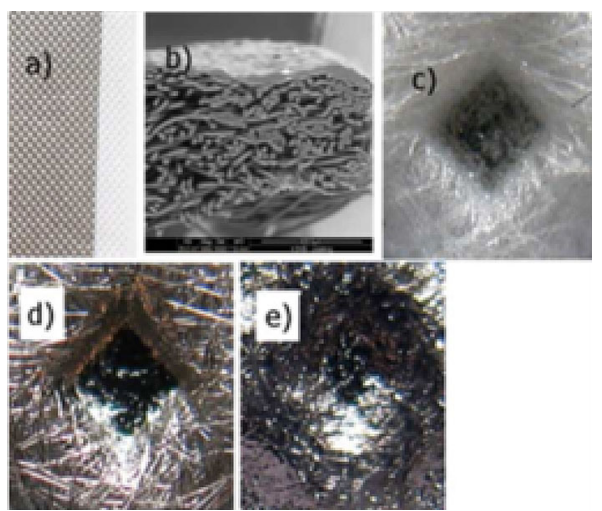

Fig. 1. Microscopic surface morphology of PP/Me composite: (a) surface of nonwoven with Me (metal) layer (grey) and without (white), (b) cross-section of composite, (c) welding point of nonwoven, (d) welding point of nonwoven with Me layer, (e) as in (d) with increasing power emitted on sputtered electrode.

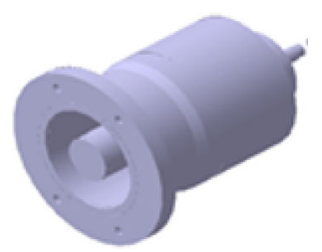

Fig. 2. Measuring adapter for SE measurement according to ASTM D4935.

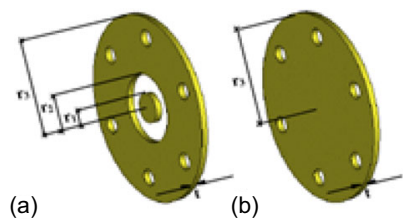

Fig. 3. Samples required shape for SE measurements: (a) reference sample, (b) load sample.

the thickness of the layers obtained - resulting in a decrease in resistivity (increase in conductivity).

\section{Shielding effectiveness}

Measurements of shielding attenuation were realised on the test setup prepared in accordance with the method of ASTM D4935-99. The test setup consists of network analyser - model E5061A ENA-L and measuring adapter, which is a section of an air concentric line of characteristic impedance $50 \mathrm{Ohm}$ (Fig. 2). Measured uncertainty is approximately $2 \mathrm{~dB}$. Cut-off frequency for this design is $1.8 \mathrm{GHz}$. Shielding attenuation is calculated as a difference between transmittances or between attenuation of a reference and an examined sample. The reference sample is in the form of a $33-\mathrm{mm}$ circle inside a $133 / 76-\mathrm{mm}$ ring (Fig. 3a), the load sample is in the form of $133 \mathrm{~mm}$ circle (Fig. 3b).

Taking into account the wave theory one can explain the EM field damping effect by the discrepancy between 


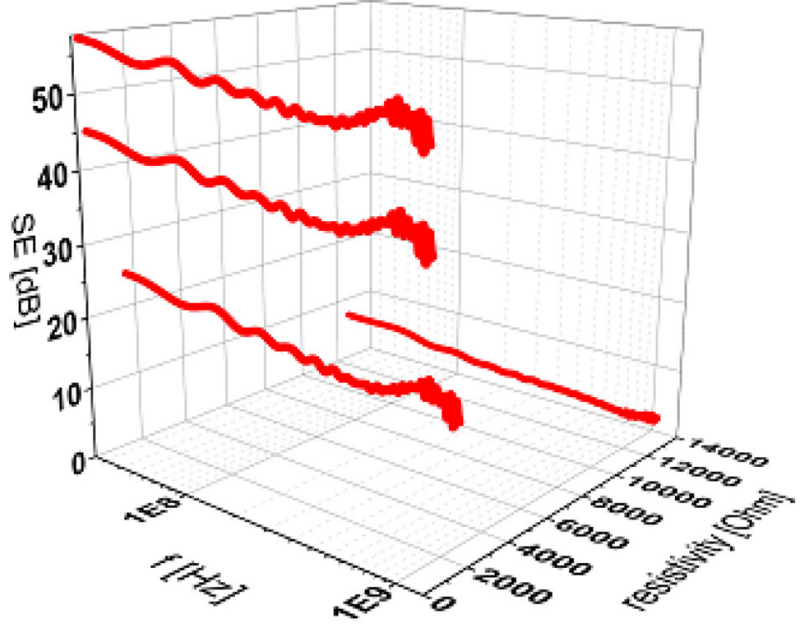

Fig. 4. Shielding effectiveness (SE) of nonwoven/Me composite.

the wave impedance of the shield and the surrounding environment [12]. When the surface of the shield has conducting properties then its wave impedance has a relatively low value, even for large frequencies of the EM wave in comparison with the wave impedance characterizing the surrounding. A decrease in the surface resistivity of the shield is therefore connected with an increase of the SE coefficient. In the case of nonwoven fabric containing conductive layer, conductive connection of the layer units is not necessary, although such connection reinforces screening properties [13]. Deposition of thin plasma layer on the rough fabric surface should also increase the active surface of the shield, which strongly influences the effectiveness of reflection at interfacial layers. The obtained values of the SE coefficient are closely related to the surface resistivity of the produced metallic layers, reaching a value of to almost $60 \mathrm{~dB}$ (Fig. 4). Nonwoven electromagnetic shields with carbon layers were characterized by low efficiency of screening, not exceeding $10 \mathrm{~dB}$. This was due to a high resistivity surface layers of carbon, because of their diamond-like structure [14].

Despite of the initial assumption, that the surface resistivity may be a good tool for testing composites $\mathrm{PP} / \mathrm{Me}$ (Me- metal) for evaluation of their shielding properties, it has been found, that deciding role is played by the thickness and uniformity of layers. In case of composites $\mathrm{PP} / \mathrm{Zn}$ and $\mathrm{PP} / \mathrm{Ti}$, for the same $\rho_{s}$, different values of shielding coefficient were obtained (Fig. 5). In this case, $\mathrm{Zn}$ layer is characterized by higher thickness and uniformity than Ti layer. Higher uniformity and continuity of Zn layers causes increase in the reflecting effect of electromagnetic wave from this surface.

\section{Dielectric characterization}

Shielding effectiveness of composite material polymermetal is not only determined by its surface resistivity. Also physical phenomena occur both in each composite

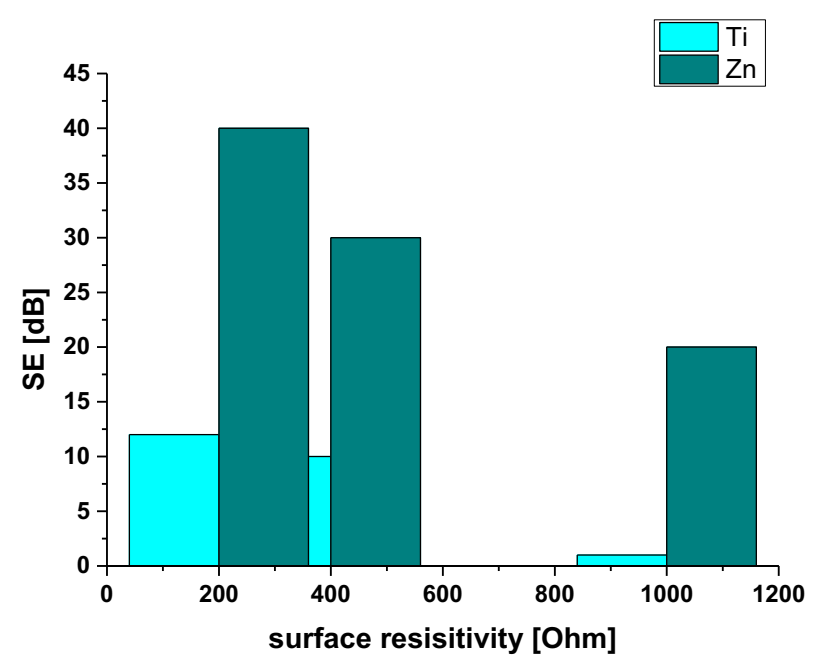

Fig. 5. Shielding effectiveness (SE) of nonwoven covered by different metals.

layer and on interphases between them perform a very important role.

Composite materials working in alternating electrical fields are characterised by a presence, beside conductivity, also polarisation phenomena [15]. The total dielectric polarisation is a superposition of three polarisation mechanisms:

- distortion polarisation, which occurs both in dipole dielectrics and in non-dipole dielectrics, and being a sum of:

- electron polarisation resulting from a deformation of electron shell, and

- atomic polarisation resulting from a displacement of atoms from their original positions,

- orientation (dipole) polarisation, which occurs only in dielectrics with permanent dipole moments resulting from an alignment of dipole axes along direction of outer electric field lines,

- ion polarisation, which occurs in dielectrics with ion bonds, resulting from displacement of ions in relation to each other from the place of balance.

Generally the polarisation phenomenon is that an arising on a dielectric surface, inserted into electric field, induced electric charges, which are bonded charges $\rho_{p}$. If we take into consideration the surface charge density for a capacitor not filled with dielectric $\rho_{0}$, then the total charge density for a capacitor with dielectric is a sum of the bounded charge $\rho_{p}$ and the free charge $\rho_{0}$.

Inserting of dielectric into electric field (flat capacitor configuration) causes increasing of the capacitance from value $C_{0}$ to value $C$. The ratio of the capacitor capacitance increasing to its capacitance $C$ is defined as the electric susceptibility of the dielectric $\chi$, but the proportion of the capacitor capacitance with a dielectric to its capacitance without a dielectric, as the electrical permittivity $\varepsilon$. Thus we can define the density of the total capacitor charge as:

$$
\rho=\rho_{0}+\rho_{p}=\rho_{0}+\chi \rho_{0}=\rho_{0}(\chi+1) .
$$


A measure of this charge is the dielectric displacement vector (the vector of an electrical flux density, the induction vector) $D$ :

$$
D \cdot n=\rho=\rho_{0}(\chi+1)=\varepsilon_{0} \cdot E \cdot n+P \cdot n,
$$

where: $n$ - is the normal unit vector directed in the dielectric direction; $\varepsilon_{0}-$ is permittivity of vacuum.

Polarisation $P$, directly bonded with surface density of polarisation charge $\rho_{p}$, we can therefore present in the form:

$$
\begin{aligned}
P & =D-\varepsilon_{0} E=(\chi+1) \varepsilon_{0} E-\varepsilon_{0} E=\varepsilon \varepsilon_{0} E-\varepsilon_{0} E \\
& =(\varepsilon-1) \varepsilon_{0} E=\chi \varepsilon_{0} E .
\end{aligned}
$$

The fundament of the impedance spectroscopy method (IS) is measurement of a linear electrical answer of examined material to an excitation with a relatively small voltage signal of the sinusoidal form $u(t)=U m \sin (\omega t+\psi u)$, in a wide frequency range $f(\omega=2 \pi f)$. An answer to the sinusoidal voltage signal is the sinusoidal form of current $i(t)=\operatorname{Im} \sin (\omega t+\psi i)$ with the same angle speed $\omega$.

This current is a sum of conductive current and polarisation (displacement) current:

$$
\begin{aligned}
i(t) & =\rho_{0} E(t)+\partial D(t) / \partial t \\
& =\rho_{0} E(t)+\varepsilon_{0} \partial E(t) / \partial t+\partial P(t) / \partial t .
\end{aligned}
$$

Using the Fourier transform to both sides of equation (9) we get an answer of dielectric in the frequency domain:

$$
\begin{aligned}
\underline{I}(\omega) & =\rho_{0} E(\omega)+j \omega \\
\underline{D}(\omega) & =\left\{\rho_{0}+\varepsilon_{0} \omega \chi^{\prime \prime}(\omega)+j \omega \varepsilon_{0}\left[1+\chi^{\prime}(\omega)\right]\right\} \underline{E}(\omega)
\end{aligned}
$$

where: $\chi^{\prime} i \chi^{\prime \prime}$ are respectively the real and the imaginary component of the complex electric susceptibility $\chi$.

Based on these measures the spectral transmittance is calculated $T(\omega)$, which characterises dependence between the forcing and the answer phase displaced for the same pulsation $\omega$ :

$$
\underline{T}(\omega)=|\underline{T}(\omega)| \cdot e^{j \psi(\omega)}
$$

where: $|\underline{T}(\omega)|$ is a modulus and $\psi(\omega)$ is an argument of the spectral transmittance $\underline{T}(\omega)$.

In the impedance spectroscopy, the spectral transmittance usually has a form of the complex impedance $Z(\omega)$ or admittance $Y(\omega)$, which are defined as:

$$
\begin{aligned}
|\underline{T}(\omega)| & =\underline{Z}(\omega)=\frac{\underline{U}(\omega)}{\underline{I}(\omega)}=|\underline{Z}(\omega)| e^{j \phi(\omega)} \\
& =\operatorname{Re} \underline{Z}+j \operatorname{Im} \underline{Z}=Z^{\prime}(\omega)+j Z^{\prime \prime}(\omega), \\
|\underline{T}(\omega)| & =\underline{Y}(\omega)=\underline{Z}^{-1}(\omega)=\frac{1}{Z} e^{-j \varphi(\omega)}=Y e^{-j \varphi} \\
& =\operatorname{Re} \underline{Y}+j \operatorname{Im} \underline{Y}=Y^{\prime}(\omega)+j Y^{\prime \prime}(\omega),
\end{aligned}
$$

where:

$$
\begin{aligned}
|\underline{Z}| & =Z=\sqrt{\left(Z^{\prime}\right)^{2}+\left(Z^{\prime \prime}\right)^{2}},|\underline{Y}|=Y=\sqrt{\left(Y^{\prime}\right)^{2}+\left(Y^{\prime \prime}\right)^{2}}, \\
\phi(\omega) & =\operatorname{arctg} \frac{Z^{\prime \prime}(\omega)}{Z^{\prime}(\omega)} .
\end{aligned}
$$

Table 1. Description of samples made of titanium and titanium monoxide layers alternately deposited on a textile.

\begin{tabular}{ccccccc}
\hline & $\mathrm{Me}$ & $\mathrm{MeO}$ & $\mathrm{Me}$ & $\mathrm{MeO}$ & $\mathrm{Me}$ & $\mathrm{MeO}$ \\
\hline$m_{3}$ & $\bullet$ & $\bullet$ & $\bullet$ & $\bullet$ & $\bullet$ & \\
\hline$m_{2}$ & & $\bullet$ & $\bullet$ & $\bullet$ & & \\
\hline$m_{1}$ & & $\bullet$ & $\bullet$ & $\bullet$ & $\bullet$ & $\bullet$ \\
\hline$m_{0}$ & $\bullet$ & & & & & \\
\hline
\end{tabular}

In the IS method we are not limited only to frequency analysis of impedance or admittance of examined material (their amplitude and phase characteristics or dependence real and imaginary parts of the complex transmittance upon frequency), but we can also use other quantities, such as the complex capacitance $C$, or the complex permittivity $\varepsilon$. These quantities are bonded with the complex admittance by frequency and geometrical parameters of sample.

The complex admittance, who can be associated a simple equivalent scheme in a parallel connection configuration of two ideal elements-resistance $R$ and capacitance $C$, is explained by a formula:

$$
\underline{Y}=\frac{1}{R}+j \omega C=G+j \omega C .
$$

Transformation of expression (6) lets to introduce a term of the complex capacitance $\underline{C}$ :

$$
\underline{C}(\omega)=\frac{\underline{Y}(\omega)}{j \omega}=C^{\prime}-j C^{\prime \prime}
$$

If we assume, that examined material of thickness $d$ is placed between two flat parallel electrodes of area $S$, that is the geometrical capacitance of capacitor electrodes configuration $C_{o}=\varepsilon_{o} S / d$ is given, then a knowledge of this parameter lets to define the relative permittivity in a complex form:

$$
\underline{\varepsilon}(\omega)=\underline{C}(\omega) / C_{o}=\underline{C}(\omega) d / \varepsilon_{o} S=\varepsilon^{\prime}-j \varepsilon^{\prime \prime} .
$$

The capacitance and the relative permittivity in a complex form are the most often used form of the spectral transmittance in the IS method. Their frequency analysis lets to show many phenomena, which take place simultaneously in an examined material.

For these examinations the measure system consist of the precise impedance analyser Agilent 4294A and the measuring cell Agilent 16451B forming the three-electrode measuring capacitor were used.

Analysis of the obtained results can be made the complex quantity presented as a function of frequency (Bode diagram) or on the complex plane (Cole-Cole diagram) as it jointly shown on Figure 6. This figure describes the dielectric properties of the composite of nonwoven plasma-coated Me (Metal)-MeO (Metal-Oxide) (Tab. 1). The analysis suggests a possibility of use to describing of polarisation phenomena, the equivalent scheme consisted of a parallel connection RC and also a presence 


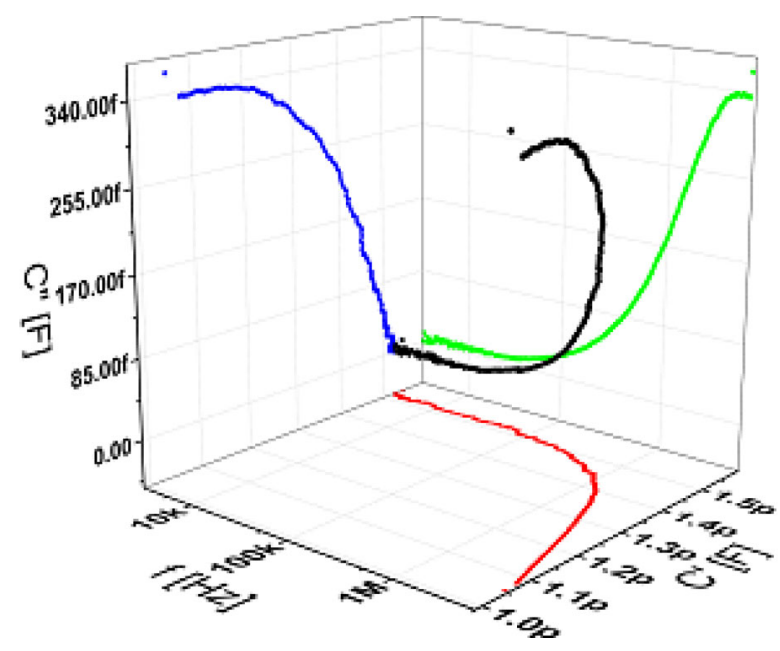

Fig. 6. Frequency spectrum of complex capacitance.

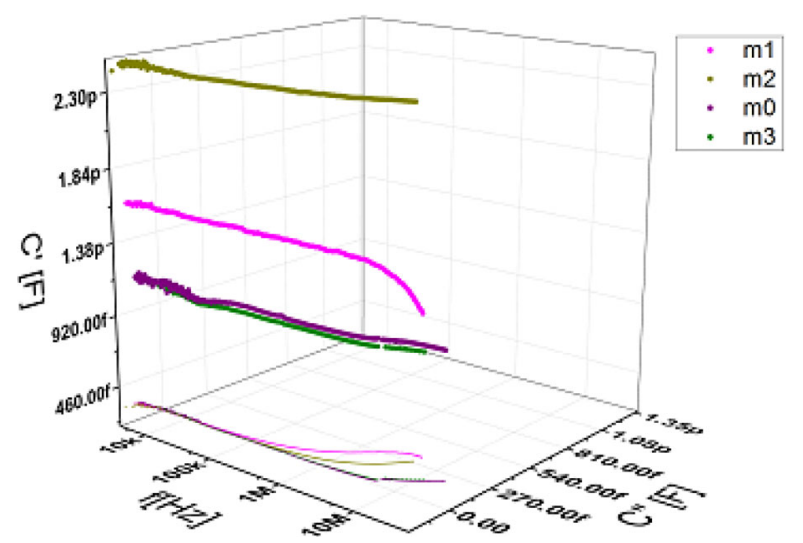

Fig. 7. Frequency spectrums of complex capacitance for PP nonvowen/Me-MeO composite.

of the Maxwell-Wagner polarisation. It is possible to find analogies to examinations outcomes some laminar structures [16].

For screens made of a nonwoven fabric with alternately arranged layers of $\mathrm{Me}$ and $\mathrm{MeO}$ (Tab. 1), one can notice some general rules [15]. We can observe a clear separation between dielectric properties of composites, in which the outer layer formed by metal (sample $m_{0}$ ), and of composites with the outer layer formed by a metal oxide (sample $m_{1}$ ) (Fig. 7). For composites with outer metal layer ( $m_{0}$ and $m_{3}$ samples) in the measuring frequency range, we observe very little differences in frequency spectrums of measured parameters and in the Cole-Cole diagrams independently from a number of $\mathrm{Me}-\mathrm{MeO}$ layers. Quite a different situation for composites with the outer layer formed by a metal oxide ( $m_{1}$ and $m_{2}$ samples) appears. In this case we can observe the strong dependence of dielectric composite properties upon a number of formed it Me-MeO layers.

The IS method has been also used to determine the connection between surface structure of a fabric being a

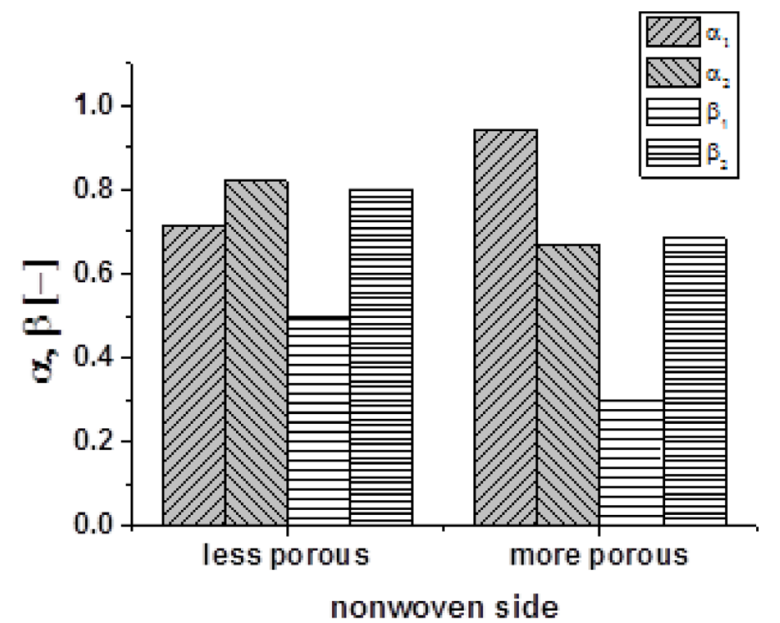

Fig. 8. Havriliak-Negami shape parameters for different nonvowen surface structures.

substrate and dielectric properties of obtained composite [17]. Relaxation processes observed in the case of the fabric with plasma layer deposited on less porous base are characterized by a much smaller amplitude and are moved in the direction of low frequencies. For samples with plasma layer deposited on more rough fabric surface in the same conditions of layer creating process, the peak exhibits considerably greater amplitude and is slightly shifted towards higher frequencies. This relaxation behavior has been described through relaxation function introduced by Havriliak and Negami:

$$
\begin{aligned}
\varepsilon^{*}(\omega) & =\varepsilon^{\prime}-i \varepsilon^{\prime \prime}=\frac{\varepsilon_{0}-\varepsilon_{\infty}}{\left[1+(i \omega \tau)^{\alpha}\right]^{\beta}}+\varepsilon_{\infty} \\
& =\frac{\Delta \varepsilon}{\left[1+(i \omega \tau)^{\alpha}\right]^{\beta}}+\varepsilon_{\infty},
\end{aligned}
$$

where: $\Delta \varepsilon$ is the relaxation strength, $\varepsilon_{0}$ is the static permittivity $\left(\varepsilon_{0}=\lim _{\omega \rightarrow 0} \varepsilon^{\prime}(\omega)\right), \varepsilon_{\infty}$ is the high-frequency limit of the permittivity $\left(\varepsilon_{\infty}=\lim _{\omega \rightarrow \infty} \varepsilon^{\prime}(\omega)\right), \alpha$ and $\beta$ are parameters describing the symmetric and asymmetric broadening of the relaxation time distribution, $\tau$ is characteristic relaxation time.

The values of the shape parameters $\alpha$ and $\beta$ (HavriliakNegami approximation), shown in Figure 8, indicate that the loss spectra of the second from observed relaxation processes are more symmetric $\left(\beta_{2} \gg \beta_{1}\right)$ but in both cases of relaxations the loss spectra of fabric with layer deposited on more porous side are more asymmetric, In the case of the fabric with layer deposited on more porous base a broader distribution of the time constants is describing a second from observed relaxation processes (lower values of parameter $\alpha_{2}$ than in the case of less porous bases).

By increasing the efficiency of the plasma layer application process, we can reduce the resistance of the surfacemodified nonwoven. It can be assumed that it is the result of the gradual elimination of discontinuities produced layer in the form of resistive bridges. 
The IS method also appears to be useful to evaluate the correlation between dielectric response of the system and surface resistance [18]. Frequency dependence of complex capacitance of composites obtained with different plasma process intensity, in the range of test frequency, indicate the presence of a single relaxation process. Increasing the intensity of the plasma process leads to a decrease of the high frequency component of the real part of the complex capacitance. In the case of samples for which found a sharp increase in the conductivity of the surface, which was accompanied by a sudden decrease in volume resistivity, the observed changes in the frequency response at high frequencies $(>0.1 \mathrm{MHz})$ suggest the presence of a second relaxation phenomenon.

\section{Summary}

In the light of the latest experiences it seems that the future in the area of EM field shielding is connected with the application of modern technologies to fabricate thin-film composite coatings. The materials are capable to fulfil all conditions of effective shielding from EM fields and eliminate all undesired occurrences associated with operation of the shielded systems. The results of our investigations, presented above, point out the possibility of industrial fabrication of the composite shielding materials with the coefficient of shielding efficiency exceeding $50 \mathrm{~dB}$. Good mechanical properties and high resistance to environmental effects are additional advantages of such materials.

Conducted research and collected experience, allowed to determine the relationship between the broadband dielectric properties of the nonwoven-plasma layer composite and: the plasma process parameters, the material being sputtered, the morphology of the substrate surface, a number of different kind of plasma layers and their order, obtained values of shielding effectiveness factor SE.

The possibilities to design desired electric properties of composite materials are also used to improve the shielding properties of the materials. Thus, the connection of the impedance spectroscopy method with those properties. It should be noted that impedance spectroscopy used as a tool to evaluate the composite materials for electromagnetic shielding allow you to solve many problems and further development work to improve the effectiveness of screening.

\section{References}

1. F. Sarto, M.S. Sarto, M.C. Larciprete, C. Sibilia, Rev. Adv. Mater. Sci. 5, 329 (2003)

2. M.S. Sarto, R. Li Voti, F. Sarto, M.C. Larciprete, IEEE Trans. EMC 47, 602 (2005)

3. L.B. Wang, K.Y. See, J.W. Zhang, B. Salam, A.C.W. Lu, IEEE Trans. EMC 53, 700 (2011)

4. J. Ziaja, M. Ozimek, J. Janukiewicz, Przeglad Elektrotechniczny 86, 222 (2010)

5. M. Jaroszewski, J. Pospieszna, J. Ziaja, G. Szafran, J. Koprowska, Przeglad Elektrotechniczny 84, 270 (2008)

6. M.L. Gulrajania, D. Gupta, Indian J. Fibre Textile Res. 36, 388 (2011)

7. K.H. Kale, A.N. Desai, Indian J. Fibre Textile Res. 36, $289(2011)$

8. D. Depla, S. Segers, W. Leroy, T. Van Hove, M. Van Parys, Textile Res. J. 81, 1808 (2011)

9. R. Perumalraj, B.S. Dasaradan, Indian J. Fibre Textile Res. 36, 35 (2011)

10. M.H. Al-Saleh, U. Sundararaj, Carbon 47, 1738 (2009)

11. R.B. Schulz, V.C. Plantz, D.R. Brush, IEEE Trans. ECM 30, 187 (1988)

12. J. Pospieszna, The basics of the electromagnetic shielding, in EM Shielding - Theory and Development of New Materials, edited by M. Jaroszewski, J. Ziaja (Kerala, Research Signpost, India, 2012)

13. J. Pospieszna, Przeglad Elektrotechniczny 4, 205 (2006)

14. J. Pospieszna, M. Jaroszewski, A. Henrykowski, G. Szafran, Przeglad Elektrotechniczny 88, 124 (2012)

15. J. Ziaja, M. Jaroszewski, EMI shielding using composite materials with plasma layers, in Electromagnetic waves, edited by V. Zhurbenko (InTech, Rijeka, 2011), pp. 425448

16. K. Nitsch, Application of Impedance Spectroscopy in the Study of Electronic Materials (Wroclaw University of Technology Press, 1999), in Polish

17. M. Jaroszewski, J. Pospieszna, J. Ziaja, J. Non-Cryst. Solids 356, 625 (2010)

18. J. Pospieszna, M. Jaroszewski, W. Bretuj, M. Tchórzewski, Przeglad Elektrotechniczny 86, 275 (2010)

Open Access This is an open access article distributed under the terms of the Creative Commons Attribution License (http://creativecommons.org/licenses/by/4.0), which permits unrestricted use, distribution, and reproduction in any medium, provided the original work is properly cited. 